\title{
Aplicación basada en Inteligencias Múltiples para apoyo al aprendizaje del inglés en preescolar. Multiple intelligent application to support english learners in preschool.
}

\author{
Claudia Marina Vicario Solórzano ${ }^{1}$, Alejandra Mendieta Rojas ${ }^{1}$, Pilar Gómez Miranda ${ }^{1}$, \\ marina.vicario@gmail.com, amendietaro@gmail.com,pgomezm@ipn.mx \\ ${ }^{1}$ Nodo UPIICSA de la Red de Computación \\ Grupo de Especialidad en Cómputo Educativo \\ Instituto Politécnico Nacional / Red LaTE Mx \\ Ciudad de México, México
}

\begin{abstract}
Resumen- En el contenido de este trabajo se describe la forma en la que se aplicó el enfoque de inteligencias múltiples al diseño instruccional de una aplicación que se orienta al apoyo del aprendizaje del inglés en el nivel preescolar de los CENDI del Instituto Politécnico Nacional de México.
\end{abstract}

Palabras clave: tecnología educativa, informática educativa, preescolar, inglés, inteligencias múltiples, CENDI

Abstract- The content of this work describes the way in which the multiple intelligences approach was applied to the instructional design of an application that is oriented towards the support of learning English in the pre-school level of the CENDI of the Instituto Politécnico Nacional in Mexico.

Keywords: Educational technology, educational computing, preschool, English, multiple intelligences, CENDI

\section{INTRODUCCIÓN}

Actualmente el Instituto Politécnico Nacional (en adelante IPN) cuenta con cinco Centros de Desarrollo Infantil (CENDI, 2010).

Los CENDI comprenden dos servicios de acuerdo con la edad del niño: de 45 días a un año once meses son lactantes; de dos años a cinco años once meses corresponden a preescolar (SEP, 2013:102) para los cuales el Grupo de Especialidad de Cómputo Educativo de la Red de Cómputo del IPN ha venido acompañando en su proceso de incorporación de tecnología (IPN-ESCOM, 2014), las más de las veces desarrollada a la medida, tal es el caso de la aplicación que se viene implementando para apoyar el aprendizaje de inglés; la cual retoma una metodología de aprendizaje inspirada en las inteligencias múltiples de Gardner considerando además favorecer el desarrollo del aprendizaje, competencias y habilidades fundamentales que demanda la Sociedad del Conocimiento.

\section{CONTEXTO}

El proyecto en general tuvo como objetivo:

"Producir un recurso tecno-educativo que apoye el aprendizaje del inglés para ser utilizado en dispositivos móviles, considerados como elementos estratégicos en la Reforma Educativa del nivel preescolar, con el fin de que sean utilizados en los CENDI del IPN”. Ello bajo la normatividad vigente (COCENDI, s.f.) y con el espíritu de aprender haciendo conforme a las perspectivas de las múltiples inteligencias (Bertrand, Regader, 2014):

Para lo cual se requirió:

- conformar una celda de producción (Maldonado y otros, 2013) que incluyó además del programador, un coordinador general de la producción, un especialista en el tema y el diseño instruccional y un diseñador gráfico como figuras mínimas.

- contar con la asesoría de una persona experta en el tema de la enseñanza del inglés para Preescolar que aplica el modelo de Gardner.

\section{DESCRIPCIÓN}

A partir del potencial de la celda y el objetivo para la producción del recurso, se retoman en el diseño de la aplicación y en la estrategia de su modelo de uso didáctico los siguientes elementos de la Teoría de las Inteligencias Múltiples ideada por el psicólogo Howard Gardner como contrapeso al paradigma de una inteligencia única.

\section{Inteligencia lingüística}

Es quizás la inteligencia que más se explota en el recurso dada la naturaleza de la aplicación, es decir el aprendizaje del idioma inglés es de por sí un reto a la inteligencia lingüística del sujeto, pero como se verá más adelante, no sólo en tanto se hace referencia a la habilidad para la comunicación oral que se pretende, sino particularmente por la notación pictográfica en la que se basa la estrategia didáctica (figura 2). 


\section{Inteligencia lógico-matemática}

La notación pictográfica (figura 2) vuelve a ser motivo para desarrollar esta inteligencia entrada en el razonamiento lógico pues reta a los aprendices en cuanto a la operación de reemplazo del elemento iconográfico por el componente verbal generando y poniendo en acción las conexiones neuronales requeridas para tal proceso.

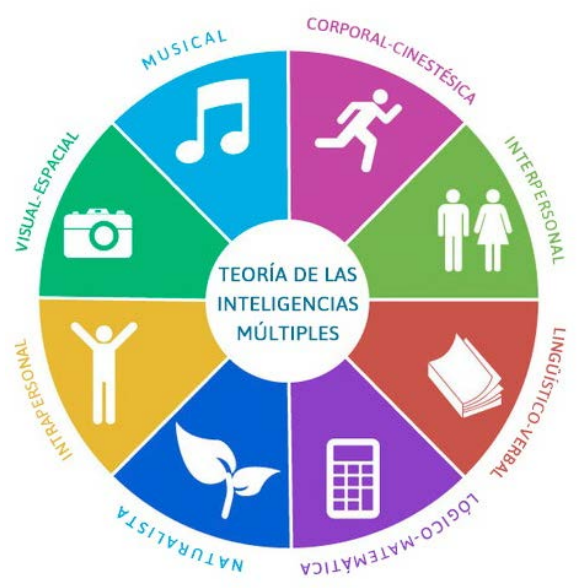

Figura 1. Las inteligencias múltiples de Gardner Fuente: Bertrand, Regader (2014)

\section{Inteligencia espacial}

Dado que el recurso va acompañado de una estrategia didáctica y ésta incluye el uso de materiales tradicionales y no sólo digitales, los niños realizarán creaciones manuales para apoyarles en el proceso de aprendizaje de los elementos nemotécnicos basados en los pictogramas del software. Esto es, se les da a colorear tales componentes y a utilizarlos como material físico 3D para expresarse en capacidad creativa también visual y estética propia de esta inteligencia.

\section{Inteligencia musical}

De igual manera la inteligencia musical, quedó latente en la aplicación justo a través de una actividad de Karaoke que permite que al usarlo los niños estimulen esta capacidad y por tanto, que algunas zonas del cerebro ejecuten funciones vinculadas con la interpretación en este ámbito, de acuerdo con el autor.

\section{Inteligencia corporal y cinestésica}

$\mathrm{Al}$ igual que con la inteligencia espacial, en la estrategia didáctica de uso del Karaoke se prevé que los niños muevan el cuerpo a manera de baile tanto en forma libre como coordinada facilitándoles que expresen incluso emociones durante la actividad. Además, el uso de los dispositivos móviles que involucra la aplicación también refieren un estímulo a dicha inteligencia.

Manteniéndose en todo momento la definición científica de la inteligencia, como la «capacidad de solucionar problemas o elaborar bienes valiosos» con la que el mismo Gardner no entró en contradicción (Bertrand, Regader, 2014)
De acuerdo a la estrategia didáctica utilizada por la profesora Gabriela Manzano, y apoyados del Programa Oficial de Inglés para el nivel (SEP, 2011) el diseño instruccional de la aplicación se organizó de la siguiente manera:

\section{Fase 1. Presentación}

Donde se presenta un pequeño diálogo entre un niño o niña (se elegirá al inicio) y la profesora.

- Niño(a): Hello. My name is

- Niño(a): This is my school. This is my classroom.

- Niño(a): She is my teacher.

- Profesora: Good morning

- Niño(a): Fine, thank you. How are you?

Adicional al audio, y con el fin de facilitar la lectura del diálogo de los niños, la aplicación utiliza algunos pictogramas como los siguientes:

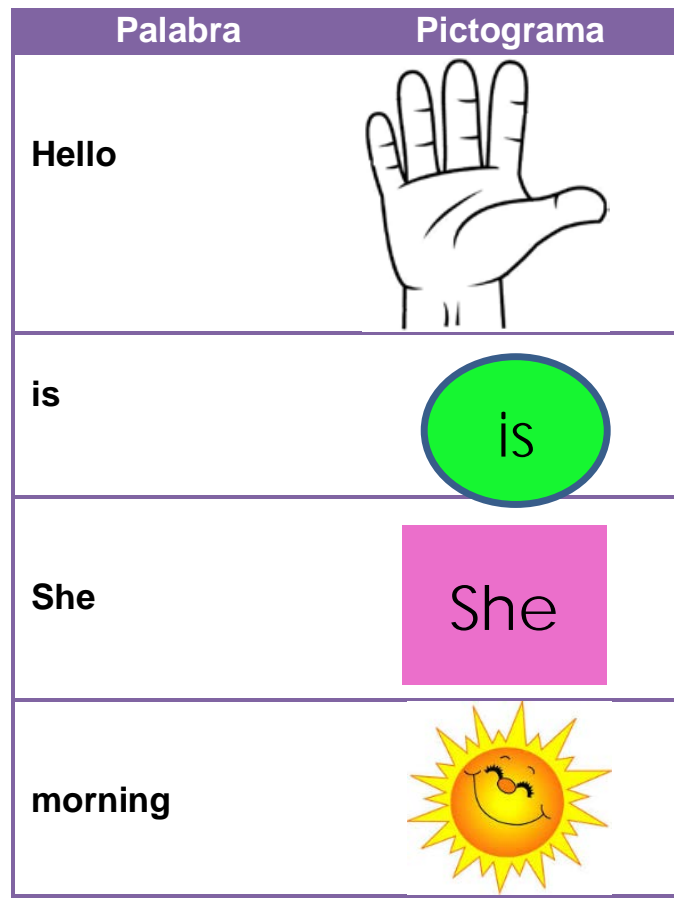

Figura 2. Pictogramas de apoyo al inglés Fuente: Manzano, Gabriela (2015)

En la segunda parte se realiza la presentación de algunos objetos que se encuentran en el salón de clases, dando su nombre y su color, por ejemplo:

- This is my Paint. It is blue.

- This is my crayon. It is pink.

- This is my color. It is purple. 


\section{Fase 2. Práctica}

Después de que los niños en la primera fase conocen el vocabulario, la siguiente fase consiste en que practiquen lo que aprendieron y para ello se diseñó la siguiente actividad:

- Se muestra la imagen de uno de los objetos del vocabulario que se enseñó, después el niño o niña (según lo que hayan elegido en la primera fase) realiza una pregunta como la siguiente:

$$
\text { - Is it my crayon? }
$$

- Posteriormente, el alumno tiene que dar clic en uno de los dos botones que aparecerán (respuesta positiva o negativa), si la respuesta que da es correcta, se le mostrará una carita feliz, en caso contrario, una carita triste.

Por último, los niños tendrán en la aplicación una canción, que podrán cantar como si fuera un karaoke, esta canción se adaptó de tal manera que se utilizará parte del vocabulario aprendido:

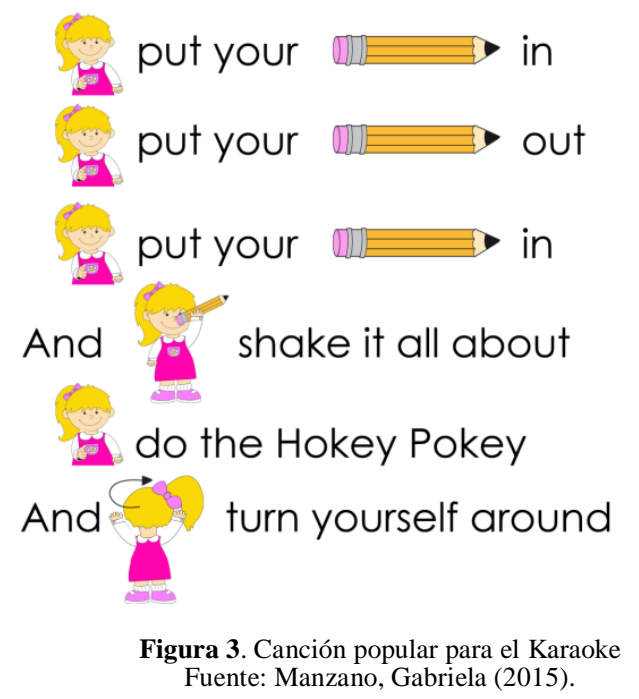

\section{Resultados}

Una vez que se contó con el diseño instruccional para el recurso, en la lógica de Berger, C. y Kam, R. (1996), y la grabación de las frases y canciones, se comenzó con su desarrollo, el cual consistió en una página web con la siguiente funcionalidad:

El menú principal de la página tiene tres iconos con la siguiente funcionalidad:

\section{Presentation \\ 2. Practice \\ 3. Production}

Presentation tiene como objetivo mostrar todo el vocabulario, para ello se muestra la imagen, la oración correspondiente y así mismo, se reproduce el audio de dicha oración.

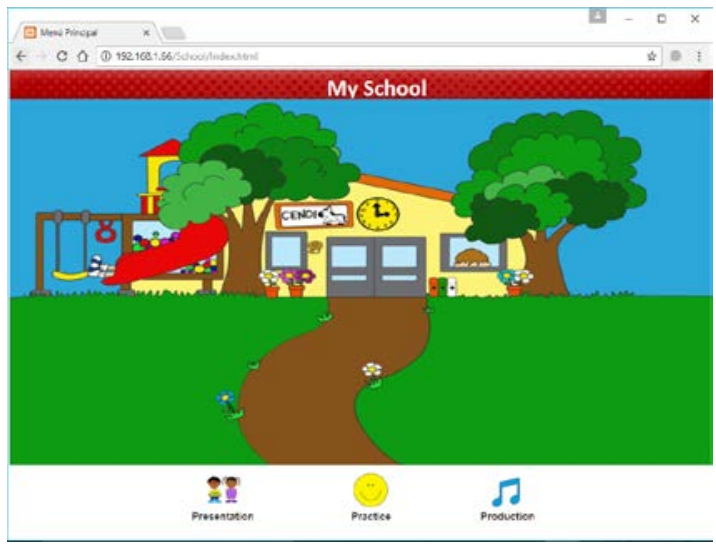

Figura 4. Pantalla principal Fuente: Desarrollo propio

Practice tiene como objetivo evaluar el aprendizaje del vocabulario mostrado en Presentation, esto a través de un test que está dividido en 5 niveles:

- $\quad$ Nivel 1: tiene un total de 5 preguntas.

- Nivel 2: tiene un total de 10 preguntas.

- Nivel 3: tiene un total de 15 preguntas.

- $\quad$ Nivel 4: tiene un total de 20 preguntas.

- $\quad$ Nivel 5: tiene un total de 25 preguntas.

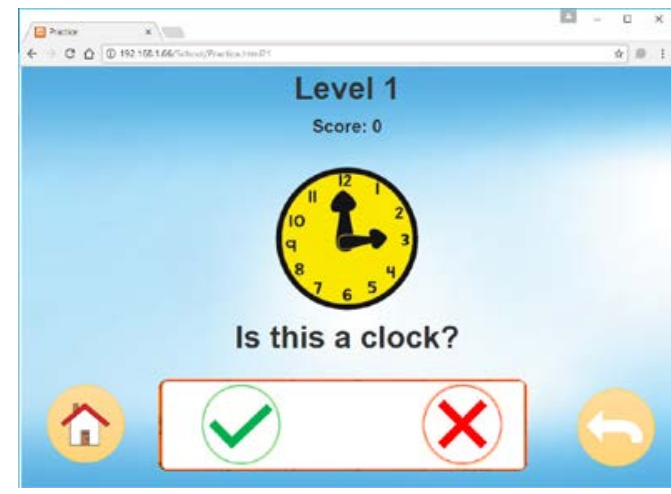

Figura5. Pantalla de la función Practice Fuente: Desarrollo propio

El último módulo llamado Production, muestra un video tipo Karaoke con la canción de Hockey Pockey:

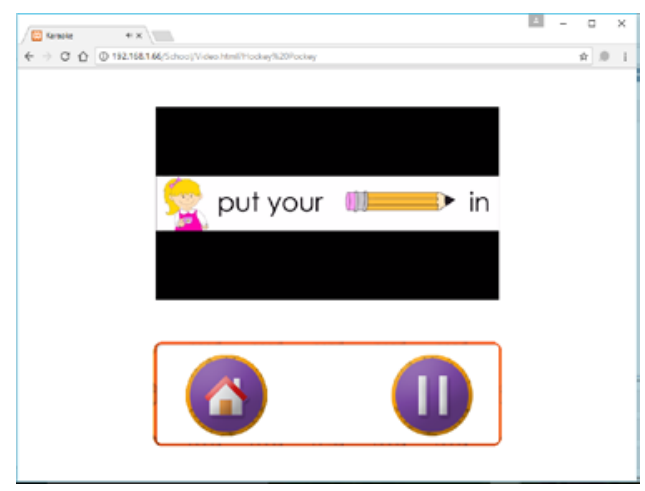

Figura 6..Pantalla de la función de Production Fuente: Desarrollo propio 
La etapa final del proyecto, hasta el punto de la producción comprometida para este ciclo escolr, consistió en realizar exclusivamente pruebas de la funcionalidad del Recurso Educativo desarrollado, ya que se espera realizar ejercicios piloto para valorar aspectos netamente cognitivos para el ciclo escolar 2017-2018 a iniciar plenamente e septiembre del 2017 con apoyo de la Red LaTE Mx.

Para ello, se tomó como referencia a Pérez, García y Hernández (2015) quienes exponen los siguientes pasos a seguir para realizar pruebas de software con niños:

1. Especificaciones de la prueba: se determina tipo y número de usuarios, guión de las pruebas, así como las tareas a realizar por cada usuario.

2. Implementación: se determina lugar, condiciones y recursos a utilizar.

3. Evaluación: análisis de los resultados obtenidos los cuales permiten conocer el nivel de usabilidad que alcanza el prototipo actual del sistema e identificar los fallos de usabilidad existentes.

En cuanto a la guía de las pruebas se definió lo siguiente:

1. Conectar los dispositivos móviles que serían utilizados para las pruebas a una misma red, así como la laptop que tomaría el papel de servidor.

2. Probar la funcionalidad del Menú principal.

3. Probar la funcionalidad del módulo Presentation.

4. Probar la funcionalidad el módulo Practice.

5. Probar la funcionalidad del módulo Production.

Se determinó utilizar a 5 niños de cada nivel de Preescolar, pues el objetivo es que dicho recurso esté disponible en algún sitio y pueda ser utilizado por los niños de los 3 niveles.

Tabla 1.. .Muestra para la fase de pruebas

\begin{tabular}{|c|l|l|c|c|}
\hline \multicolumn{2}{|c|}{ Edad } & Nivel & $\begin{array}{c}\text { Total } \\
\text { niñas }\end{array}$ & $\begin{array}{c}\text { Total } \\
\text { niños }\end{array}$ \\
\hline Grupo 1 & 3 años & Preesc. 1 & 2 & 3 \\
\hline Grupo 2 & 4 años & Preesc. 2 & 2 & 3 \\
\hline Grupo 3 & 5 años & Preesc. 3 & 2 & 3 \\
\hline \multicolumn{5}{|c|}{ Fuente: Desarrollo propio } \\
\hline
\end{tabular}

Antes de comenzar las pruebas, se hizo una pequeña entrevista a los niños, para conocer su experiencia previa en el uso de los dispositivos móviles y computadoras, resultando que todos los niños han utilizado en su mayoría el smartphone y la Tablet, siendo las computadoras las menos utilizadas.

A partir de ello se probó la funcionalidad de los tres servicios: Presentation, Practice y Production con los tres grados.

Al finalizar las pruebas de todos los módulos, se realizó un pequeño cuestionario a los niños, con el fin de analizar su experiencia con el uso del Recurso Educativo Digital "La Escuela”, obteniendo los siguientes resultados:
- El $100 \%$ de los niños indicó que les gustó la aplicación.

- El 93.33\% de los niños calificó el recurso con 5 estrellitas (siendo 5 el número más alto).

- $\quad$ El 27\% de los niños indicó que el recurso fue difícil de utilizar, mientras el 73\% indicó que estuvo fácil.

- Se solicitó que indicaran qué fue les pareció lo más difícil por lo que:

o El $47 \%$ de los niños contestaron que el juego.

o El $47 \%$ de los niños contestaron que nada fue difícil.

o El 6\% de los niños contestaron que el vocabulario.

- En cuanto a lo que más les gustó las respuestas fueron:

o El $47 \%$ de los niños contestaron que la canción.

o El 33\% de los niños contestaron el juego

o El 13\% de los niños contestaron que el vocabulario, el juego y la canción.

o El 7\% de los niños contestaron que el vocabulario.

- Finalmente, se preguntó qué fue lo que aprendieron al utilizar el recurso, por lo que sus respuestas fueron:

o El $80 \%$ de los niños contestaron que nuevo vocabulario.

o El 13\% de los niños contestaron que la canción.

o El 7\% de los niños contestaron que nada.

\section{CONCLUSIONES}

Analizando el desarrollo de las pruebas y los resultados obtenidos, se concluyó que un recurso tecno-educativo para apoyo al aprendizaje de otra lengua desde las primeras edades que, como éste orientado al inglés, retome elementos de la Teoría de las Inteligencias múltiples de Gardner, se convierte en una herramienta útil y divertida para favorecer el aprendizaje, aunque con algunas diferencias en cuanto a las habilidades digitales asociadas, según la edad; ya que se observó particularmente que:

- Después de que los niños comenzaron a utilizar el Recurso Educativo, algunos comenzaban a repetir las frases en inglés de manera espontánea e imitando la misma entonación.

- Los niños de Preescolar 2 y 3 entendieron más rápido la funcionalidad del recurso.

- Algunos niños aprendieron nuevo vocabulario.

- La canción fue lo que más les gustó y les causaba más diversión.

- Los niños de Preescolar 3 pudieron explorar el recurso por sí mismos. 
- Los niños fueron capaces de identificar imágenes y relacionarlas con su entorno.

- Los niños de Preescolar 3 identificaron lo que representaba cada estrellita (acierto o desacierto), mientras los niños de Preescolar 1 y 2 no prestaron atención en eso.

Por otro lado también es posible señalar algunos puntos que no resultaron favorables durante las pruebas y que pueden considerarse a futuro para anticipar algún quiebre como en nuestro caso :

El lugar elegido por la maestra Manzano para realizar las pruebas (el patio escolar) no fue el más adecuado, ya que provocaba que nos niños se distrajeran fácilmente. Se sugiere utilizar el aula de clase normal.

La conexión a internet fue muy lenta, por lo que se optó por utilizar una red de celular. Conviene por tanto asegurar una buena conectividad.

Se reitera que el alcance del proyecto hasta hoy estuvo centrado en el diseño instruccional, la producción y pruebas de funcionalidad del Recurso, de modo que los aspectos de transferencia y pruebas piloto con enfoque psicopedagógico son líneas de trabajo con alcances a Julio del 2018.

Finalmente compartimos con la comunidad internacional que investiga y desarrolla Tecnología Educativa nuestra convicción de la importancia de hacer investigación a partir de acciones de producción de recursos propios, como en este caso, ya que ello favorece también el desarrollo de la ingeniería y la ciencia para estos campos de conocimiento en nuestros países (Vicario, Argüelles, Jalife, Rodríguez, 2016).

\section{Agradecimientos}

Las autoras del presente trabajo agradecen a la COCENDI del IPN por las facilidades para la realización de la aplicación y en particular a la Profesora Gabriela Manzano Torres por compartir su experiencia en la aplicación de la metodología de inteligencias múltiples en la enseñanza del inglés y su apoyo, incondicional, en la elaboración de los guiones didácticos y supervisión del recurso interactivo en la fase de pruebas.

Así mismo agradecemos y damos todos los créditos al INSTITUTO POLITÉCNICO NACIONAL de México ya que este artículo deriva de los apoyos otorgados para el Proyecto de Investigación con carácter Multidisciplinario denominado "Servicios de Tecnología Educativa de última generación, basados en buenas prácticas e ITIL para la Red LaTE Mx" con clave SIP 1899 del Instituto Politécnico Nacional y sus módulos asociados: SIP-20170350, SIP-20170375, SIP20170362 y SIP-20170364. Junto con las facilidades para las grabaciones y edición de audios de los niños por parte de ONCETV México.

\section{REFERENCIAS}

COCENDI (2010): Lineamientos para la Operación y Funcionamiento de los Centros de Desarrollo Infantil del Instituto Politécnico Nacional. Consultado el 3 de marzo de 2017.2 Disponible en http://www.cocendi.ipn.mx/Documents/lineamientosCO CENDI_gaceta2012.pdf

COCENDI (s.f.): Programa de Educación Inicial (PEI). Consultado el 8 de marzo de 2017. Disponible en http://www.cocendi.ipn.mx/Conocenos/Paginas/Modelo _Educativo.aspx

Berger, C. y Kam, R. (1996): Definitions of Instructional Design. Adapted from "Training and Instructional Design". Applied Research Laboratory, Penn State University. Consultado el 8 de mayo de 2014. Disponible http://www.umich.edu/ ed626/define.html

Bertrand, Regader (2014): Psicología y Mente. La Teoría de las Inteligencias Múltiples de Gardner. Consultado el 20 de junio de $2015 . \quad$ Disponible en: http://psicologiaymente.net/la-teoria-de-lasinteligencias-multiples-de-h-gardner/

Blázquez, Florentino; Lucero, Manuel (2002). Los medios y recursos en el proceso didáctico. En Medina, A. \& Salvador, F. Didáctica General (pp. 185- 218). Madrid: Pearson Educación

IPN-ESCOM (2014): Programa de Desarrollo Informático Educativo de los CENDI IPN. Consultado el 13 de febrero de 2014. Disponible en: https://proyectosip20140338.wordpress.com/informe/

Manzano, Gabriela (2015): Estrategias para la enseñanza de inglés. Consultado el 15 de julio de 2015.

Maldonado, Jorge; Mejía, Magali; Muñoz Lissette (2013): Propuesta Metodológica Para La Creación De Material Educativo Digital. Consultado el 26 de octubre de 2016. Disponible

http://www.virtualeduca.org/ponencias2014/207/Propues taMetodologicaparalacreaciondeMED.docx

Pérez, María E.; García, Mónica; Hernández, Jorge A. (2015): Consideraciones especiales para realizar pruebas de usabilidad con niños: Caso de Estudio., ReCIBE. Consultado el 29 de Junio de 2017. Disponible en: http://recibe.cucei.udg.mx/revista/es/vol4no1/pdf/computacion02.pdf

Rosales, Ysabel (2013): Aprender haciendo: Metodología de elaboración de software educativo y recursos digitales. Consultado el 19 de marzo de 2017. Disponible en http://www.virtualeduca.info/ponencias2012/210/Ysabel RosalesChicnesMetodologiadedesarrolodesoftwareeduca tivoyrecursosdigitales.doc

SEP a) (2013): Programa Sectorial de Educación 2013-2018. Consultado el 26 de marzo de 2017. Disponible en http://www.sep.gob.mx/work/models/sep1/Resource/447 9/4/images/PROGRAMA_SECTORIAL_DE_EDUCAC ION_2013_2018_WEB.pdf 
SEP (2013): Programa de Estudio Preescolar, Consultado el 15 de septiembre 2017. Disponible en http://www.curriculobasica.sep.gob.mx/index.php/progpreescolar1/prog-est-prees-programa

SEP (2011): Programa Nacional de Inglés en Educación Básica, Consultado el 13 de septiembre 2017. Disponible en

http://www.curriculobasica.sep.gob.mx/pdf/pnieb/pnieb_ fundamentos.pdf
SEP (2013): Educación Preescolar, SEP. Consultado el 23 de marzo de 2017. Disponible en http://www2.sepdf.gob.mx/que_hacemos/preescolar.jsp

Vicario, Marina, Argüelles, Amadeo, Jalife, Salma y Rodríguez, Teresa (2016) coordinadores: Breve análisis del estado del conocimiento y perspectivas de la Industria de la Tecnología Educativa en México, Red LaTE México-IPN-CONACYT, CUDI, México. 